\title{
MDCT evaluation of aortic root and aortic valve prior to TAVI. What is the optimal imaging time point in the cardiac cycle?
}

\author{
Tomas Jurencak • Jakub Turek • Bastiaan L. J. H. Kietselaer • Casper Mihl • \\ Madeleine Kok • Vincent G. V. A. van Ommen • Leen A. F. M. van Garsse • \\ Estelle C. Nijssen • Joachim E. Wildberger • Marco Das
}

Received: 4 July 2014 / Revised: 7 December 2014 / Accepted: 15 January 2015 / Published online: 24 February 2015

(C) The Author(s) 2015. This article is published with open access at Springerlink.com

\begin{abstract}
Objectives To determine the optimal imaging time point for transcatheter aortic valve implantation (TAVI) therapy planning by comprehensive evaluation of the aortic root.

Methods Multidetector-row CT (MDCT) examination with retrospective ECG gating was retrospectively performed in 64 consecutive patients referred for pre-TAVI assessment. Eighteen different parameters of the aortic root were evaluated at 11 different time points in the cardiac cycle. Time points at which maximal (or minimal) sizes were determined, and dimension differences to other time points were evaluated. Theoretical prosthesis sizing based on different measurements was compared.

Results Largest dimensions were found between 10 and $20 \%$ of the cardiac cycle for annular short diameter (10\%); mean diameter $(10 \%)$; effective diameter and circumferencederived diameter $(20 \%)$; distance from the annulus to right coronary artery ostium (10\%); aortic root at the left coronary
\end{abstract}

Tomas Jurencak and Jakub Turek contributed equally to this work.

T. Jurencak · J. Turek · B. L. J. H. Kietselaer · C. Mihl • M. Kok • E. C. Nijssen · J. E. Wildberger • M. Das $(\square)$

Department of Radiology, Maastricht University Medical Center,

P. Debyelaan 25, P.O. Box 5800, 6202, AZ Maastricht,

The Netherlands

e-mail: m.das@mumc.nl

B. L. J. H. Kietselaer · C. Mihl • M. Kok · J. E. Wildberger • M. Das CARIM School for Cardiovascular Diseases, Maastricht University Medical Center, Maastricht, The Netherlands

B. L. J. H. Kietselaer • V. G. V. A. van Ommen

Department of Cardiology, Maastricht University Medical Center,

Maastricht, The Netherlands

L. A. F. M. van Garsse

Department of Cardiothoracic Surgery, Maastricht University

Medical Center, Maastricht, The Netherlands artery level (20\%); aortic root at the widest portion of coronary sinuses $(20 \%)$; and right leaflet length $(20 \%)$. Prosthesis size selection differed depending on the chosen measurements in $25-75 \%$ of cases.

Conclusion Significant changes in anatomical structures of the aortic root during the cardiac cycle are crucial for TAVI planning. Imaging in systole is mandatory to obtain maximal dimensions.

Key Points

- Most aortic root structures undergo significant dimensional changes throughout the cardiac cycle.

- The largest dimensions of aortic parameters should be determined to optimize TAVI.

- Circumference-derived diameter showed maximum dimension at $20 \%$ of the cardiac cycle.

Keywords Aortic valve $\cdot$ Aortic valve stenosis $\cdot$ Multidetector computed tomography $\cdot$ Cardiac gated imaging technique . Heart valve prosthesis implantation

$\begin{array}{ll}\text { Abbreviations } \\ \text { AASR } & \text { aortic annulus sphericity ratio } \\ \text { AVA } & \text { aortic valve area } \\ \text { CIN } & \text { contrast-induced nephropathy } \\ \text { CM } & \text { contrast media } \\ \text { LBBB } & \text { left bundle branch block } \\ \text { MDCT } & \text { multidetector computed tomography } \\ \text { MPRs } & \text { multiplanar reformations } \\ \text { TAVI } & \text { transcatheter aortic valve implantation }\end{array}$

\section{Introduction}

Transcatheter aortic valve implantation (TAVI) provides a minimally invasive therapeutic option for patients with aortic 
valve stenosis who cannot undergo conventional open-heart surgery [1-5]. Pre-TAVI evaluation requires precise measurement of the aortic root and aortic valve. Unfortunately, current prostheses still impose annular size limitations [6,7]. As the aortic root is a complex anatomical structure which changes dynamically throughout the cardiac cycle, besides the aortic annulus other anatomical structures (e.g. the sinotubular junction) should be considered for TAVI planning as well. Possible complications, resulting from wrong prosthesis choice or difficulties encountered during placement, may result in paravalvular regurgitation, valve embolization, coronary obstruction, left bundle branch block (LBBB) or aortic annular rupture [8-10].

Multidetector-row CT (MDCT) is the method of choice in pre-TAVI assessment of aortic annulus, aortic root and ascending aorta $[11,12]$ showing superiority over other modalities [13]. Depending on the chosen CT scan mode, MDCT offers the additional possibility of complex evaluation of the aortic root and aortoiliac vasculature within one acquisition $[14,15]$. High spatial and temporal resolutions of MDCT allow comprehensive imaging of the aortic root, and the possibility of three-dimensional multiplanar reformations (MPRs) gives unique viewing and measuring options. Recently, MDCT measurements were shown to be predictive factors for the severity of paravalvular leakage in TAVI patients, thereby further supporting for the use of MDCT in pre-TAVI assessment $[16,17]$.

Aortic annular size plays a key role in TAVI valve size selection. Its measurement, however, is complicated by the fact that the aortic annulus undergoes dynamical changes in systole and diastole in patients, whether with diseased or non-diseased aortic valves [18, 19]. Recently, de Heer et al. [18] used postprocessing software to analyse dynamical changes of the aortic annulus by evaluating area and radius changes during the cardiac cycle, and found significant changes in these parameters. Other studies measuring aortic dimensions using MDCT have used different time points in the cardiac cycle, such as systolic images [6] or diastolic images [20], or images without ECG triggering [21, 22].The differing methods in themselves will result in different dimensions for the same structure. Although it is yet unclear whether annular dimension changes described in the literature are clinically relevant [23], one may assume that measurements derived from the time point at which a structure is at its largest are unlikely to lead to size underestimation and wrong prosthesis choice.

Current TAVI valve sizing recommendations are based on annulus dimension (mainly diameter), but many more parameters are available and can be assessed as to which might play a role in TAVI sizing and TAVI valve design. Yet there is a lack of data regarding temporal dimensional changes of other structures within the aortic root, which might play an important role in TAVI planning and outcome. The optimal imaging time point will have a large impact on the CT protocol used, since large differences exist between protocols in radiation dose and contrast media (CM) bolus required. The latter is especially relevant to the TAVI patient population in view of susceptibility to contrast-induced nephropathy (CIN).

The aim of this study was to determine the optimal imaging time point within the cardiac cycle for the measurement of critical structures of the aortic root in pre-TAVI assessment, and to provide information on the assessment of aortic annulus shape changes during the cardiac cycle.

\section{Material and methods}

Patient population

Sixty-four consecutive patients with severe symptomatic aortic stenosis and referred to our institution for pre-TAVI assessment were retrospectively evaluated between November 2010 and February 2012. Patients received a pre-interventional MDCT scan for the assessment of aortic root and valve dimensions, including an assessment of peripheral arteries. The patient sample included 27 men and 37 women, mean age was $81.3 \pm 5$ years. Heart rate during image acquisition was recorded. As a result of the retrospective nature of this study, waiver of informed consent was obtained through the local ethical committee (METC14-4-027).

Image acquisition

Subjects underwent retrospectively ECG-gated spiral MDCT of the aortic valve region (Somatom Definition Flash, Siemens, Forchheim, Germany). The CT protocol was as follows: tube voltage $100 \mathrm{kV}$, tube current $320 \mathrm{mAs}$, pitch 0.23 , temporal resolution $75 \mathrm{~ms}$, collimation $2 \times 2 \times 64 \times$ $0.6 \mathrm{~mm}$. The CM injection protocol included $120 \mathrm{ml}$ of CM (Iopromide, Ultravist 300, Bayer, Berlin, Germany): $20 \mathrm{ml}$ of $\mathrm{CM}$ and $15 \mathrm{ml}$ of saline were injected as a test bolus, followed by $75 \mathrm{ml}$ pure CM, $50 \mathrm{ml} 50 \% \mathrm{CM}$ solution and finally a 50 $\mathrm{ml}$ saline flush, all injected at a rate of $7.2 \mathrm{ml} / \mathrm{s}$. Images were reconstructed $(0.75 \mathrm{~mm}$ slice thickness, $0.7 \mathrm{~mm}$ increment, B26f kernel) at 11 time points of the R-R interval: at $20 \mathrm{~ms}$ after the $\mathrm{R}$ wave and at every $10 \%$ of the $\mathrm{R}-\mathrm{R}$ interval.

\section{Measurements}

Two research fellows specially trained in TAVI evaluation took measurements independently. For final analysis, consensus was reached for each measurement. Measurements were taken at each of the 11 time points mentioned above, using dedicated software syngo.via (Siemens Healthcare, Forchheim, Germany). Aortic annulus was assessed in an oblique transversal plane crossing the most basal attachments of the aortic leaflets. An oblique transversal plane 
perpendicular to the course of the aorta was used for the measuring of dimensions of the aortic root at the level of sinotubular junction, right and left coronary artery ostium, and for the measurement of the widest portion of coronary sinuses. The oblique sagittal or coronal plane was subsequently used for the measurement of the distances between the coronary artery ostium centre and aortic annulus, and the maximal length of the right and left aortic leaflets.

The following measurements of the aortic root and the aortic valve were performed using MPRs according to the Society of Cardiovascular Computed Tomography (SCCT) expert consensus guidelines [24] (see also Fig. 1): (1) short annulus diameter; (2) long annulus diameter; (3) area (defined as an oval or ring formed by linking the most basal portions of the leaflet attachments); (4) annulus circumference; (5) distance from the aortic annulus to the centre of the ostium of the right coronary artery; (6) distance from the aortic annulus to the centre of the ostium of the left coronary artery; (7) sinotubular junction diameter; (8) aortic root diameter at the level of the right coronary artery ostium; (9) aortic root diameter at the level of the left coronary artery ostium; (10) widest portion of the coronary sinuses diameter; (11) left aortic valve leaflet length and (12) right aortic valve leaflet length, including distances from the leaflet basal insertion to the free edge.

The mean diameter (13) was defined as the average of short and long diameters of an aortic annulus; circumference-derived diameter (14) was defined as circumference/ $\pi$; and the effective diameter (15) was defined by the following formula [25]:

$E D=2^{*} v\left(\right.$ cross $^{-}$sectional area $\left._{p} / p\right)$
The following parameters were used in the assessment of annulus shape: aortic annulus sphericity ratio (16) $(\mathrm{AASR}=$ long diameter/short diameter; an AASR of 1 implies a perfect circular shape); ellipse eccentricity (17) as defined by the following formula [26]:

$\varepsilon=\sqrt{\left[1-(\text { short } / \text { long })^{2}\right]}$

(If $\varepsilon=0$, the ellipse is a circle); difference between long and short diameters (18) expressed in absolute values (millimetres) and percentages. If the difference between long and short diameter was at least $3 \mathrm{~mm}$, the shape of the aortic annulus was considered an oval. All dimensions were measured by two independent observers.

Prosthesis size was determined on the basis of minimal and maximal measurements of mean diameter, circumferencederived diameter and effective diameter, according to recommendations by the various vendors: Sapien Valve XT (Edwards Lifesciences Corp, Irvine, USA), CoreValve (Medtronic, Minneapolis, USA) and JenaValve (JenaValve, Munich, Germany). The resulting prosthesis choices were compared in order to deduce how often chosen valve sizes differed between methods used.

\section{Statistical analysis}

Dimensions of each anatomical structure at each time point were evaluated using descriptive statistics (mean \pm standard deviation; SPSS, version 19.0). Subsequently, the time point
Fig.1 Examples of particular measurements of aortic root and aortic valve dimensions: a the aortic annulus; $\mathbf{b}$ the annulus to the right coronary artery ostium; c the annulus to the left coronary artery ostium; $\mathbf{d}$ the length of the right coronary leaflet
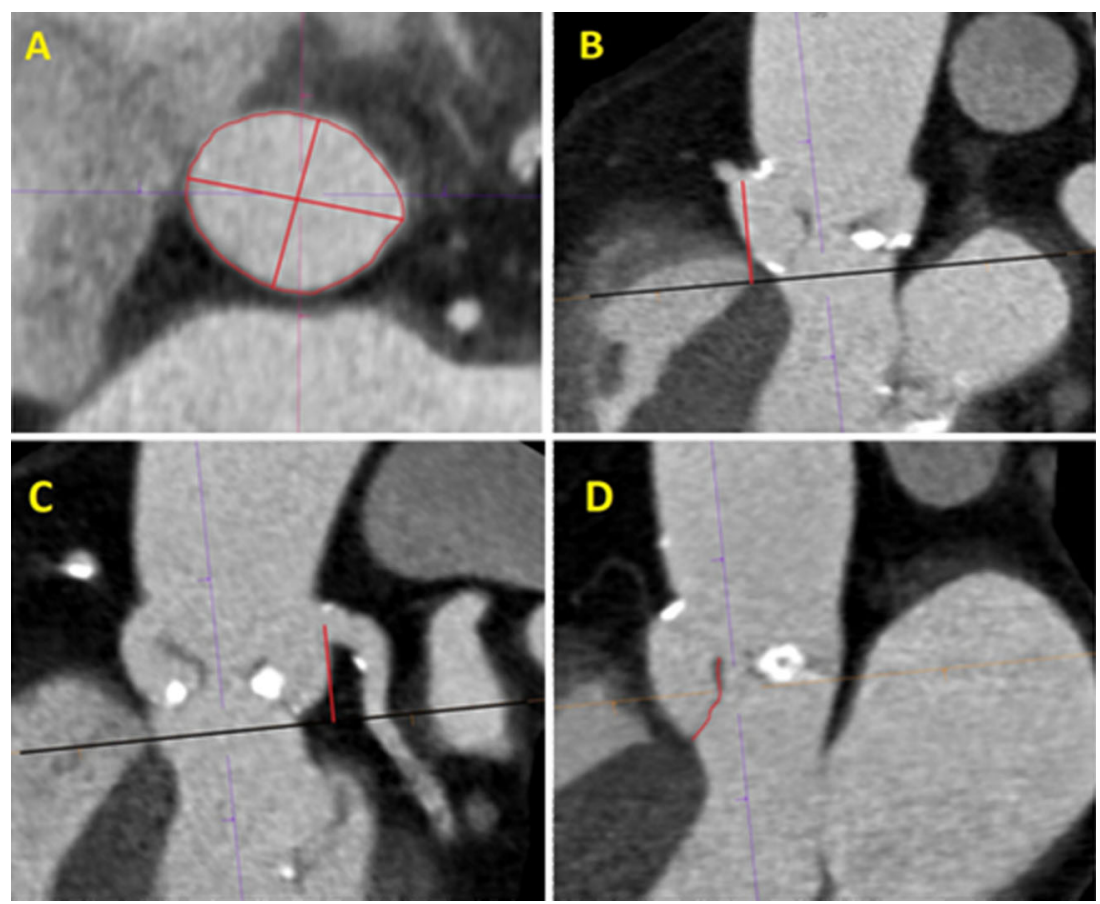
Table 1 Pre-TAVI patient characteristics as estimated by cardiac echography

\begin{tabular}{lccc}
\hline & Mean \pm SD & Min & Max \\
\hline Age (years) & $81.3 \pm 5.0$ & 63 & 89 \\
Logistic EuroSCORE $^{\mathrm{a}}$ & $18.1 \pm 8.6$ & 1.9 & 39.5 \\
Ejection fraction (\%) & $54.3 \pm 10.0$ & 25 & 69 \\
AVA (cm ${ }^{2}$ ) & $0.8 \pm 0.2$ & 0.4 & 1.0 \\
$\begin{array}{l}\text { Aortic stenosis (max pressure } \\
\text { gradient, mmHg) }\end{array}$ & $74.6 \pm 23.8$ & 25 & 138 \\
$\begin{array}{c}\text { Aortic stenosis (mean pressure } \\
\text { gradient, mmHg) }\end{array}$ & $45.3 \pm 15.5$ & 12 & 88 \\
\hline
\end{tabular}

$A V A$ aortic valve stenosis

${ }^{\text {a }}$ Logistic EuroSCORE used to estimate cardiac operative risk

at which the biggest or smallest dimension was determined and compared to those obtained at other time points using a paired-samples $t$ test. The maximal mean values of diameter, circumference-derived diameter and effective diameter were compared using a paired-samples $t$ test. Statistically significant differences $(p<0.05)$ were investigated further. Kappa analysis was performed to test interobserver agreement. Data analysis was conducted with SPSS version 20.0 (SPSS Inc, Chicago, IL, USA).

\section{Results}

General patient characteristics are summarized in Table 1. No patients with aneurysm or bicuspid valve or genetic disorders were included. MDCT with retrospective ECG gating provided images of sufficient quality to allow measurement of all anatomical structures included in this study. Mean heart rate of the patient during scan acquisition was $69 \mathrm{bpm}$ (SD 8). Mean radiation dose was DLP 555 (mGy cm) (SD 225). Table 2 presents the dimensions found for the anatomical structures examined: mean, maximal and minimal dimensions with standard deviations, corresponding time points, and the number of time points at which dimensions were significantly different from the maximum or minimum $(p<0.05)$. Almost perfect interobserver correlation was found for all parameters (0.87). The lowest interobserver correlation was found for the length of the right cusp (0.77) and the highest was found for the annulus short axis diameter (0.91).

Table 2 Overview of examined anatomical structures: maximum and minimum, dimensions with corresponding time points, and the number of time points at which dimensions were significantly different from the maximum or minimum

\begin{tabular}{llll}
\hline Structure & $\begin{array}{l}\text { Minimal diameter } \\
\text { (time point \%) }\end{array}$ & $\begin{array}{l}\text { Mean } \\
\text { diameter }\end{array}$ & $\begin{array}{l}\text { Maximal diameter } \\
\text { (time point \%) }\end{array}$
\end{tabular}

Number of time points at which measurements differed significantly from max. or min. values $(p<0.05)$

\begin{tabular}{|c|c|c|c|c|}
\hline 1. Annulus short diameter $(\mathrm{mm})$ & $20.9 \pm 2.0(80 \%)$ & $21.6 \pm 2.0$ & $22.6 \pm 2.0(10 \%)$ & $10(\max )$ \\
\hline 2. Annulus long diameter (mm) & $27.2 \pm 2.7(100 \%)$ & $27.4 \pm 2.4$ & $27.7 \pm 2.4(60 \%)$ & $0(\max )$ \\
\hline 3. Area $\left(\mathrm{mm}^{2}\right)$ & $417.6 \pm 62.8(50 \%)$ & $473.3 \pm 64.5$ & $467.3 \pm 67.8(20 \%)$ & $10(\max )$ \\
\hline 4. Circumference (mm) & $75.8 \pm 5.8(50 \%)$ & $77.2 \pm 6.2$ & $79.0 \pm 6.0(20 \%)$ & $10(\max )$ \\
\hline 5. Right coronary artery ostium distance $(\mathrm{mm})$ & $17.6 \pm 2.8(10 \%)$ & $17.8 \pm 2.8$ & $18.0 \pm 2.9(20 \mathrm{~ms})$ & 10 (min) \\
\hline 6. Left coronary artery ostium distance (mm) & $16.2 \pm 2.4(20 \mathrm{~ms})$ & $16.8 \pm 2.4$ & $17.4 \pm 2.2(100 \%)$ & $2(\min )$ \\
\hline $\begin{array}{l}\text { 7. Ascending aorta at the level of sinotubular junction } \\
(\mathrm{mm})\end{array}$ & $27.1 \pm 2.2(90 \%)$ & $27.4 \pm 2.4$ & $27.6 \pm 3.1(40 \%)$ & $0(\max )$ \\
\hline 8. Aortic root at right coronary artery level (mm) & $29.8 \pm 3.0(20 \mathrm{~ms})$ & $30.2 \pm 3.3$ & $30.8 \pm 3.3(40 \%)$ & $1(\max )$ \\
\hline 9. Aortic root at left coronary artery level (mm) & $32.1 \pm 3.4(50 \%)$ & $32.4 \pm 3.6$ & $33.0 \pm 3.5(20 \%)$ & $7(\max )$ \\
\hline 10. Aortic root at the widest portion of aortic sinuses (mm) & $34.1 \pm 3.5(90 \%)$ & $34.4 \pm 3.6$ & $34.9 \pm 3.6(20 \%)$ & $6(\max )$ \\
\hline 11. Left leaflet length (mm) & $15.8 \pm 2.0(10 \%)$ & $16.0 \pm 2.0$ & $16.3 \pm 1.8(60 \%)$ & $0(\max )$ \\
\hline 12. Right leaflet length (mm) & $15.4 \pm 1.7(80 \%)$ & $15.7 \pm 1.8$ & $16.1 \pm 2.0(20 \%)$ & $6(\max )$ \\
\hline 13. Mean diameter $(\mathrm{mm})$ & $23.9 \pm 2.9(70 \%)$ & $24.4 \pm 2.3$ & $25.0 \pm 2.0(10 \%)$ & $9(\max )$ \\
\hline 14. Effective diameter (mm) & $23.0 \pm 1.8(50 \%)$ & $23.5 \pm 1.8$ & $24.3 \pm 1.8(20 \%)$ & $10(\max )$ \\
\hline 15. Circumference-derived diameter (mm) & $24.1 \pm 1.9(50 \%)$ & $24.6 \pm 1.9$ & $25.2 \pm 1.9(20 \%)$ & $10(\max )$ \\
\hline 16. AASR & $\begin{array}{l}1.215 \pm 0.082 \\
(10 \%)\end{array}$ & $1.277 \pm 0.111$ & $\begin{array}{l}1.317 \pm 0.107 \\
(90 \%)\end{array}$ & $5(\max )$ \\
\hline 17. Ellipse eccentricity & $\begin{array}{l}0.554 \pm 0.082 \\
\quad(10 \%)\end{array}$ & $0.604 \pm 0.092$ & $\begin{array}{l}0.638 \pm 0.072 \\
\quad(90 \%)\end{array}$ & $5(\max )$ \\
\hline 18. Difference between Dmax and Dmin (mm) & $4.8 \pm 1.6(10 \%)$ & $5.9 \pm 2.2$ & $6.7 \pm 2.1(60 \%)$ & $5(\max )$ \\
\hline
\end{tabular}

Except for distance RCA and LM to the annulus where minimal distance is most relevant, for the other parameters maximal measure is most relevant AASR aortic annulus sphericity ratio 
For 15 of the 18 dimensions measured at least one time point was found at which the maximal value differed significantly. Half the structures measured $(9 / 18)$ were largest during systole at $10-20 \%$ of the cardiac cycle. Only three structures (aortic annulus long diameter, ascending aorta at the level of the sinotubular junction, and left leaflet length) did not show any significant dimensional changes throughout the cardiac cycle. On the other hand, annulus short diameter, circumference, area, distance from the annulus to the right coronary ostium, circumference-derived diameter and effective diameter differed significantly at all time points from maximal measurements.

The aortic annulus short diameter was largest at $10 \%$ of the $\mathrm{R}-\mathrm{R}$ interval and changed significantly throughout the whole cardiac cycle, while the aortic annulus long diameter was largest at diastolic reconstruction at $60 \%$, without undergoing significant changes throughout the cardiac cycle. Both area and annulus circumference showed maximal measurements at $20 \%$ of the $\mathrm{R}-\mathrm{R}$ interval, undergoing significant changes throughout the remaining cardiac cycle. The circumferencederived and mean diameters were biggest at $20 \%$ of the $\mathrm{R}-\mathrm{R}$ interval, changing significantly throughout the rest of the cardiac cycle. Mean diameter showed the biggest mean measurements at $10 \%$ of the cardiac cycle, but no statistically significant difference was found between the $10 \%$ and $20 \%$ time points ( $p=0.373$ ) (Fig. 2).

The mean circumference-derived diameter at $20 \%$ of the cardiac cycle differed significantly from the effective diameter at $20 \%(p<0.05)$, but not significantly from the mean diameter at $10 \%(p=0.221)$.
Where minimal dimensions were measured (i.e. distance to right and left coronary ostia) these were found during systole (right $10 \%$; left $20 \mathrm{~ms}$ ).

For both AASR and ellipse eccentricity index, the maximum was found at $90 \%$ and the minimum at $10 \%$ of the R-R interval. Compared to the maximal values, five significantly different time points were observed (20 ms, 10-30\%, $100 \%$ ), two of which were systolic. Similarly, the difference between long and short aortic annulus diameter was smallest at $10 \%$, and the maximum value differed significantly at five time points. The latter maximum value was, however, found at $60 \%$ of the cardiac cycle. The evaluation of the annular shape revealed the following values: AASR 1.28 \pm 0.11 , ellipse eccentricity $0.603 \pm 0.092$ and difference between long and short annular diameter $5.9 \pm 2.2 \mathrm{~mm}(27.8 \pm 11.3 \%$ ) (Fig. 3).

In this population 43 patients received a $23-\mathrm{mm}$ Edwards Sapien valve, 11 patients received a 26-mm Edwards Sapien valve and one patient received a JenaValve $(25 \mathrm{~mm})$. Thirtysix patients were treated with a transapical approach, three with a transaortal approach and 16 with a transfemoral approach. Nine patients finally did not undergo TAVI implantation. Differences in prosthesis size choice using either maximal or minimal measurements of mean diameter, circumference-derived diameter and effective diameter are summarized in Table 3. Edwards Sapien valve prosthesis choice would change in $25 \%$ (effective diameter) to $48 \%$ (circumference-derived diameter) of cases; CoreValve in $43.8 \%$ (mean diameter) to $48 \%$ (effective diameter) of cases; and JenaValve in $75 \%$ of cases for all three evaluated dimensions. Table 4 summarizes the percentage of patients suited for
Fig.2 Box plot showing the dimensions at each time point of four annulus structures. Red boxes represent the time point at which the largest measurements were made. Maximum measurements of all of these structures significantly differed to those at other time points in the cardiac cycle, except for mean diameter whose maximum at $20 \%$ was not significantly different to the maximal measurement at $10 \%$ of the cardiac cycle
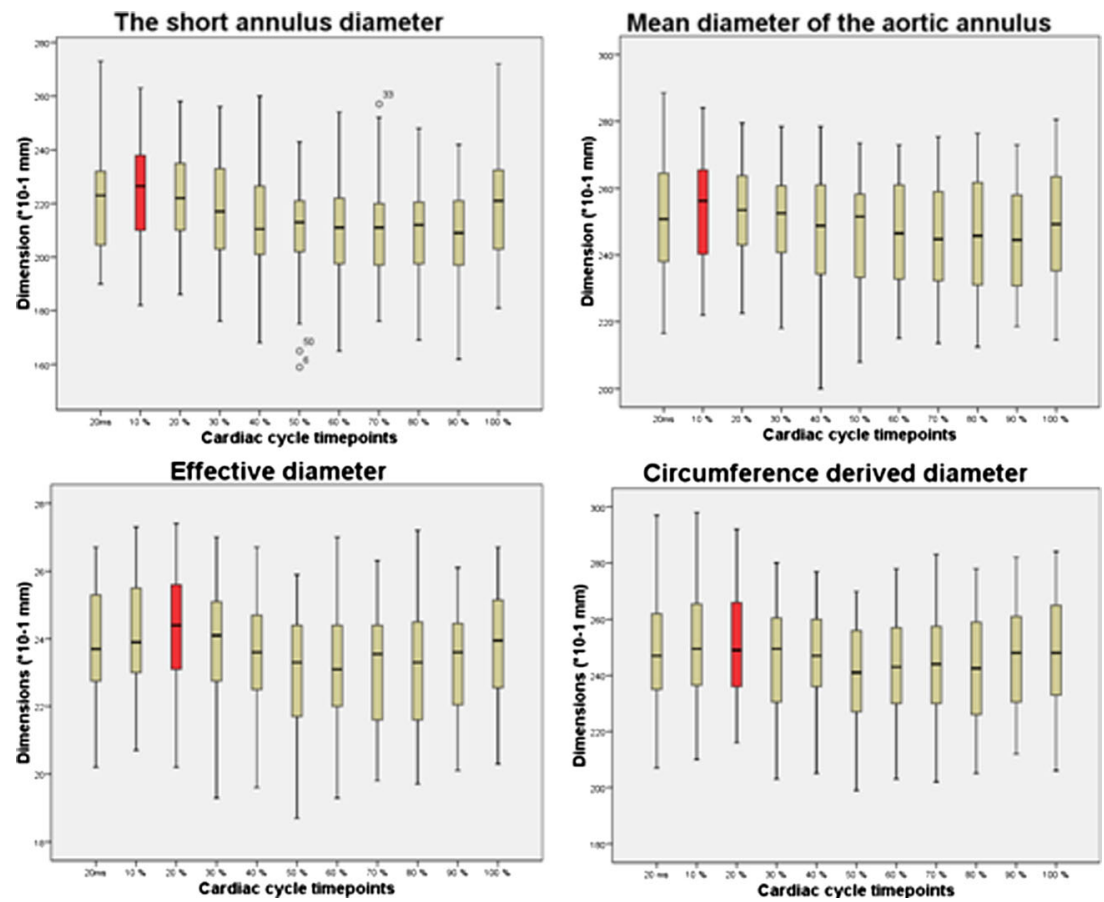
Fig.3 Changes during the cardiac cycle of mean aortic annulus sphericity ratio, ellipse eccentricity and difference between long and short diameters

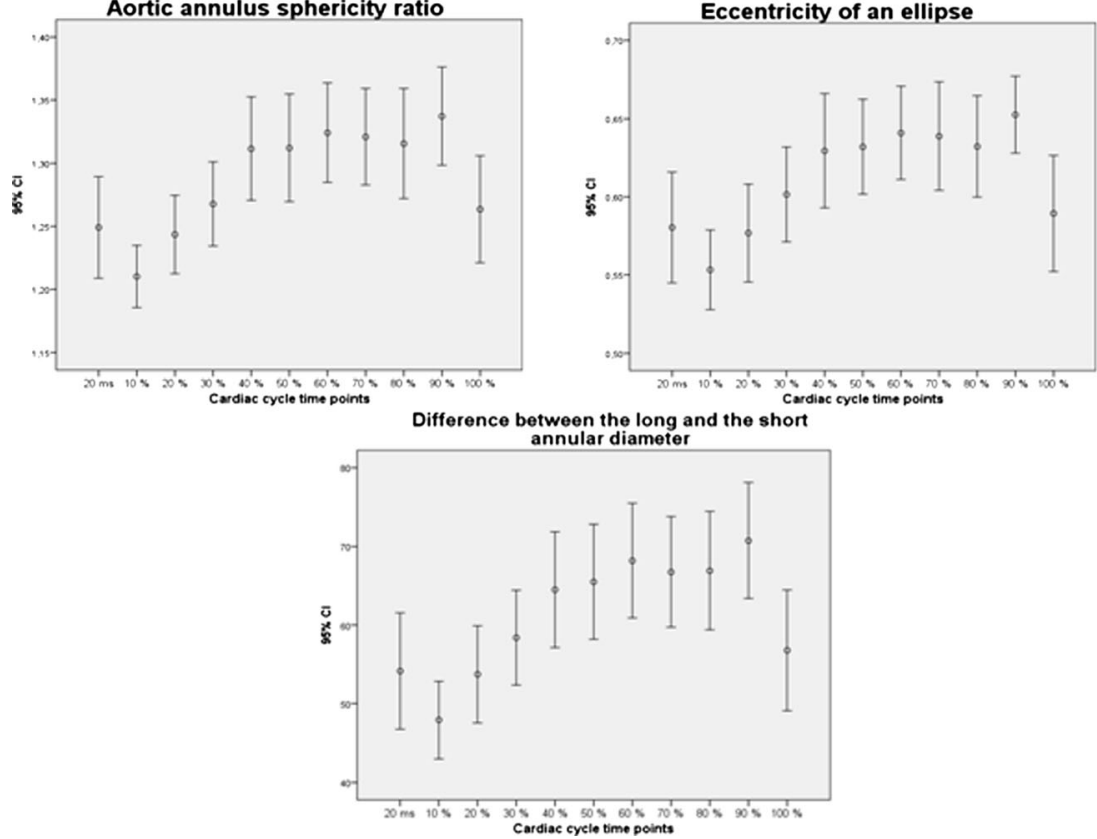

particular prosthesis selection, based on maximal and minimal diameters.

\section{Outcome data}

Seven patients developed a left bundle branch block (LBBB), three patients a right bundle branch block (RBBB). Four patients had to receive a pacemaker and seven patients developed an intraventricular conduction delay (IVCD). Five patients developed mild (10-20\%) paravalvular regurgitation, one patient severe paravalvular regurgitation $(30 \%)$.

\section{Discussion}

Observed variations in aortic root dimensions during the cardiac cycle have been reported in the literature concerning

Table 3 Differences in prosthesis size when maximal and minimal measurements of mean diameter, circumference-derived diameter and effective diameter were used

\begin{tabular}{llll}
\hline & Edwards Sapien & CoreValve & JenaValve \\
\hline Mean diameter (\%) & 40.6 & 43.8 & 75 \\
$\begin{array}{l}\text { Circumference-derived } \\
\quad \text { diameter (\%) }\end{array}$ & 48 & 46.9 & 75 \\
Effective diameter (\%) & 25 & 48 & 75 \\
\hline
\end{tabular}

Table shows what percentage of the study population would have undersized prosthesis if the time point with minimal measured diameter were used for prosthesis sizing instead of the maximal one. Official recommendations for prosthesis sizing of the particular vendors were used
Table 4 Percentage of chosen prostheses of particular sizes from different vendors based on vendors' sizing recommendations

\begin{tabular}{|c|c|c|c|c|}
\hline \multirow[b]{2}{*}{ Prosthesis size } & \multicolumn{2}{|c|}{ Mean diameter } & \multicolumn{2}{|c|}{ Circumference-derived diameter } \\
\hline & $\operatorname{Min}(\%)$ & $\operatorname{Max}(\%)$ & $\operatorname{Min}(\%)$ & $\operatorname{Max}(\%)$ \\
\hline \multicolumn{5}{|c|}{ Edwards Sapien valve } \\
\hline $20 \mathrm{~mm}$ & 0 & 0 & 0 & 0 \\
\hline $23 \mathrm{~mm}$ & 11 & 0 & 11 & 0 \\
\hline $26 \mathrm{~mm}$ & 38 & 23 & 44 & 20 \\
\hline $29 \mathrm{~mm}$ & 22 & 31 & 17 & 27 \\
\hline $23 / 26 \mathrm{~mm}$ & 14 & 5 & 9 & 5 \\
\hline $26 / 29 \mathrm{~mm}$ & 16 & 20 & 17 & 22 \\
\hline Not matched & 0 & 20 & 2 & 27 \\
\hline \multicolumn{5}{|l|}{ CoreValve } \\
\hline $23 \mathrm{~mm}$ & 2 & 0 & 6 & 0 \\
\hline $26 \mathrm{~mm}$ & 39 & 14 & 45 & 20 \\
\hline $29 \mathrm{~mm}$ & 50 & 50 & 44 & 56 \\
\hline $31 \mathrm{~mm}$ & 0 & 19 & 5 & 3 \\
\hline $23 / 26 \mathrm{~mm}$ & 2 & 0 & 3 & 0 \\
\hline $26 / 29 \mathrm{~mm}$ & 2 & 0 & 0 & 0 \\
\hline $29 / 31 \mathrm{~mm}$ & 6 & 17 & 2 & 17 \\
\hline Not matched & 0 & 0 & 0 & 0 \\
\hline \multicolumn{5}{|l|}{ JenaValve } \\
\hline $23 \mathrm{~mm}$ & 33 & 16 & 39 & 16 \\
\hline $25 \mathrm{~mm}$ & 30 & 31 & 41 & 45 \\
\hline $27 \mathrm{~mm}$ & 27 & 14 & 5 & 31 \\
\hline Not matched & 11 & 19 & 16 & 8 \\
\hline
\end{tabular}

Theoretical selection based on mean diameter and circumference-derived diameter - the largest of the commonly used dimensions in our study population 
aortic root motion in patients free of aortic valve pathology $[12,18,19,27-29]$, with maximum and minimum values found during systole and diastole respectively. MDCT studies performed in subjects with non-diseased aortic valves [12] as well as in subjects with stenotic aortic valves [19] found evidence for the aortic motion causing statistically significant dimensional changes of the aortic annulus between systole and diastole, which were confirmed in our set of TAVI candidates. However, previous studies took only aortic annulus changes into account. Although the aortic annulus is a crucial structure for TAVI planning, the whole aortic root and aortic valve should be considered to determine the correct prosthesis size and position, as well as the position of aortic leaflets towards coronary ostia after prosthesis deployment.

In pre-TAVI assessment, special attention is given to annulus evaluation, because annulus dimensions are crucial to prosthesis selection. Modern prostheses are provided in a limited number of sizes [30, 31], and prosthesis-annulus incongruity, or underestimated prosthesis size, has been addressed as a significant determinant of paravalvular aortic regurgitation [10]. Other factors such as device malpositioning and heavily calcified aortic or bicuspid leaflets have also been associated with post-procedural paravalvular regurgitation [32], which in turn has been associated with increased morbidity and mortality [33]. Placing of an "oversized" prosthesis has greatly decreased paravalvular leakage $[16,17]$ and improved prognosis, although minor paravalvular leakage after TAVI is still very common. On the other hand, systematic use of oversized valves might lead to annulus rupture or LBBB $[34,35]$. Annular rupture is a catastrophic complication of TAVI, and LBBB has been associated with an increased need for pacemaker implantation [36].
Valve prosthesis sizing according to circumference [19], mean or effective diameter measurements has been proposed [37]. In the current study, all of these dimensions showed maximal values during systole $(10 \%$, mean diameter; $20 \%$, circumference-derived diameter; $20 \%$, effective diameter) (Fig. 2). The largest measurement found was for the mean circumference-derived diameter, which was significantly larger than mean effective diameter, and not significantly different to mean diameter. Although the maximal mean value for mean diameter was found at $10 \%$ of the R-R interval, no statistically significant difference between the $10 \%$ and $20 \%$ time points was observed.

Knowledge of maximal dimensions of the aortic annulus short and long diameters, area, circumference and other portions of the aortic root would be an important benefit for TAVI planning, decreasing the number of implantations of undersized prostheses (Fig. 4). Additionally, short and long aortic annulus diameter measurements might prove to be crucial if oval-shaped prostheses are introduced into routine practice.

Considering the present data, maximal dimensions of four portions of the aortic root were seen at $20 \%$ of the cardiac cycle. These four portions are effective diameter, circumference-derived diameter and aortic root at the level of the left coronary artery and at the level of the widest portion of the coronary sinuses. Measurements of the annulus to right coronary ostium distance at $20 \%$ do not show a minimum, however, being significantly higher than the smallest measurement. Although measurements of the annulus to left coronary ostium distance do not show a minimum at $20 \%$ of the cardiac cycle; the dimensions at this time point do not differ significantly from their minimal measures. Interestingly, significant changes of aortic valve leaflet dimensions were observed.

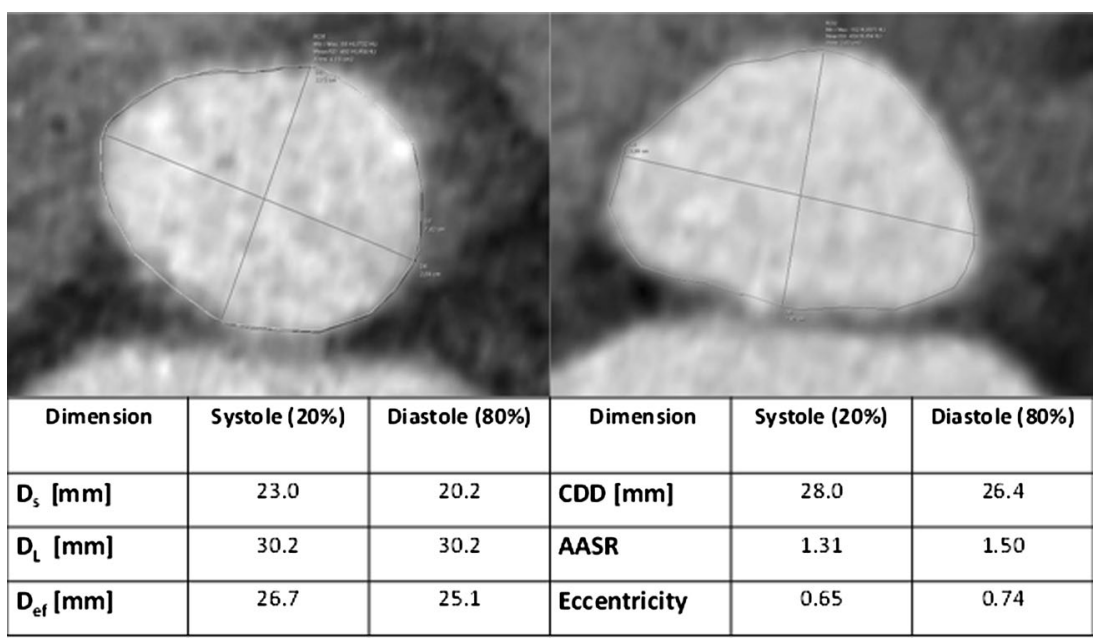

Fig.4 Example of differences in dimensions found at systolic $(20 \%)$ and diastolic $(80 \%)$ phases of the cardiac cycle. $D_{\mathrm{s}}$ annulus short diameter, $D_{\mathrm{L}}$ annulus long diameter, $D_{\text {ef }}$ effective diameter, $C D D$ circumferencederived diameter, $A A S R$ aortic annulus sphericity ratio, Eccentricity ellipse eccentricity. Using different measurements for the diameter could lead to different prosthesis sizing (e.g. annulus short axis diameter $23 \mathrm{~mm}$ at systole could lead to 26-mm Edwards valve, while the same measurement at diastole would lead to a 23-mm valve. If effective diameter were used a $29-\mathrm{mm}$ valve would be chosen) 
This could be explained by lack of accuracy due to the extensive valvular calcification often present in this cohort of patients, however.

All measurements of the aortic annulus short diameter were significantly smaller $(p<0.05)$ compared to the largest measurement at $10 \%$ of the cardiac cycle, which suggests that the aortic annulus presents the biggest dimensions in early systole where its contour resembles a more circular shape than at other cardiac phases. This agrees with the findings of previously published studies $[19,25]$. Significant differences in annular shape during the cardiac cycle were assessed by calculating different parameters (AASR, ellipse eccentricity, difference between maximal and minimal annular diameters). Our analysis showed that the aortic annulus has a persistent oval shape, with a maximum at $90 \%$. This knowledge could be useful for future prosthesis design and for the development of new aortic valve prostheses.

This study proved that significant differences among cardiac cycle time points also exist when applying the results to theoretical prosthesis selection. In the case of JenaValve prosthesis, this difference would result in $75 \%$ mismatch between maximal and minimal measurements. Assuming that prosthesis sizing based on the largest measurements prevents severe paravalvular leakage, this mismatch could have a negative influence on patient prognosis. However, several factors contribute to overall post-procedural outcomes, and these therefore need to be taken into consideration.

Our results suggest that MDCT evaluation of aortic root during early systole, namely at $10-20 \%$ of the cardiac cycle, provides the most precise measurements for TAVI planning. Presumably, valve prostheses chosen according to these measurements are unlikely to be undersized. Furthermore our data suggest that potentially not only annulus sizing should be taken into account as also other aortic root structures do show dynamic changes throughout the cardiac cycle, e.g. aortic annuls area or aortic root at the widest portion of the aortic sinuses. It is also obvious that different parameters do show different behaviour; e.g. annulus long diameter does not differ significantly between different time points but effective diameter and circumference diameter (both calculated diameters) do change at every measured time point. Furthermore annulus long diameter might not represent the optimal measurementeven though relatively stable - as it does not represent sphericity of the annulus. Therefore probably a combination of different measurements would be necessary (according to endovascular prosthesis placement in the thoracic or abdominal aorta) taking size differences of the aortic root into account.

Further studies are necessary to clarify whether procedure planning based on systolic MDCT images at $20 \%$ of the cardiac cycle contributes to better clinical results, especially to reducing the incidence of paravalvular leakage and LBBB.
Limitations

Limitations of this study include the relatively small number of patients and its retrospective nature. Furthermore, the study did not include a comparison of DSCT with other modalities, or correlations to post-interventional outcomes, and prosthesis sizing was based on theoretical assumptions.

\section{Conclusion}

The time point at $20 \%$ of the cardiac cycle provides MDCT images with maximal dimensions of structures of the aortic root and aortic valve, which are the most important for preTAVI assessment and prosthesis choice. Future prospective studies would be necessary to prove the value of other parameters presented here in optimizing valve design as well as procedure planning.

Acknowledgments The scientific guarantor of this publication is Marco Das, MD, PhD. The authors of this manuscript declare relationships with the following companies: Siemens (Forchheim, Germany), Bayer (Berlin, Germany), Philips (Best, the Netherlands). The authors state that this work has not received any funding. One of the authors has significant statistical expertise. Institutional review board approval was obtained. Written informed consent was waived by the institutional review board.

Methodology: retrospective, cross sectional study, observational, performed at one institution.

Open Access This article is distributed under the terms of the Creative Commons Attribution Noncommercial License which permits any noncommercial use, distribution, and reproduction in any medium, provided the original author(s) and the source are credited.

\section{References}

1. Cribier A, Eltchaninoff H, Tron C et al (2004) Early experience with percutaneous transcatheter implantation of heart valve prosthesis for the treatment of end-stage inoperable patients with calcific aortic stenosis. J Am Coll Cardiol 43(4):698-703

2. Grube E, Schuler G, Buellesfeld L et al (2007) Percutaneous aortic valve replacement for severe aortic stenosis in high-risk patients using the second- and current third-generation self-expanding CoreValve prosthesis: device success and 30-day clinical outcome. J Am Coll Cardiol 50(1):69-76

3. Webb JG, Chandavimol M, Thompson CR et al (2006) Percutaneous aortic valve implantation retrograde from the femoral artery. Circulation 113(6):842-850

4. Webb JG, Pasupati S, Humphries K et al (2007) Percutaneous transarterial aortic valve replacement in selected high-risk patients with aortic stenosis. Circulation 116(7):755-763

5. Svensson LG (2008) Aortic valve stenosis and regurgitation: an overview of management. J Cardiovasc Surg 49(2):297-303

6. Leipsic J, Gurvitch R, Labounty TM et al (2011) Multidetector computed tomography in transcatheter aortic valve implantation. JACC Cardiovasc Imaging 4(4):416-429 
7. Messika-Zeitoun D, Serfaty JM, Brochet E et al (2010) Multimodal assessment of the aortic annulus diameter: implications for transcatheter aortic valve implantation. J Am Coll Cardiol 55(3):186-194

8. Tamburino C, Capodanno D, Ramondo A et al (2011) Incidence and predictors of early and late mortality after transcatheter aortic valve implantation in 663 patients with severe aortic stenosis. Circulation 123(3):299-308

9. Ye J, Cheung A, Lichtenstein SV et al (2010) Transapical transcatheter aortic valve implantation: follow-up to 3 years. J Thorac Cardiovasc Surg 139(5):1107-1113, 1113 e1101

10. Detaint D, Lepage L, Himbert D et al (2009) Determinants of significant paravalvular regurgitation after transcatheter aortic valve: implantation impact of device and annulus discongruence. JACC Cardiovasc Interv 2(9):821-827

11. Smid M, Ferda J, Baxa J et al (2010) Aortic annulus and ascending aorta: comparison of preoperative and periooperative measurement in patients with aortic stenosis. Eur J Radiol 74(1):152-155

12. de Heer LM, Budde RP, Mali WP, de Vos AM, van Herwerden LA, Kluin J (2011) Aortic root dimension changes during systole and diastole: evaluation with ECG-gated multidetector row computed tomography. Int J Cardiovasc Imaging 27(8):1195-1204

13. Jilaihawi H, Kashif M, Fontana G et al (2012) Cross-sectional computed tomographic assessment improves accuracy of aortic annular sizing for transcatheter aortic valve replacement and reduces the incidence of paravalvular aortic regurgitation. J Am Coll Cardiol 59(14):1275-1286

14. Blanke P, Euringer W, Baumann Tet al (2010) Combined assessment of aortic root anatomy and aortoiliac vasculature with dual-source CT as a screening tool in patients evaluated for transcatheter aortic valve implantation. AJR Am J Roentgenol 195(4):872-881

15. Plank F, Friedrich G, Bartel T et al (2012) Benefits of high-pitch 128slice dual-source computed tomography for planning of transcatheter aortic valve implantation. Ann Thorac Surg 94(6):1961-1966

16. Buzzatti N, Maisano F, Latib A et al (2013) Computed tomographybased evaluation of aortic annulus, prosthesis size and impact on early residual aortic regurgitation after transcatheter aortic valve implantation. Eur J Cardiothorac Surg 43(1):43-50, discussion 50-41

17. Willson AB, Webb JG, Labounty TM et al (2012) 3-dimensional aortic annular assessment by multidetector computed tomography predicts moderate or severe paravalvular regurgitation after transcatheter aortic valve replacement: a multicenter retrospective analysis. J Am Coll Cardiol 59(14):1287-1294

18. de Heer LM, Budde RP, van Prehn J et al (2012) Pulsatile distention of the nondiseased and stenotic aortic valve annulus: analysis with electrocardiogram-gated computed tomography. Ann Thorac Surg 93(2):516-522

19. Hamdan A, Guetta V, Konen E et al (2012) Deformation dynamics and mechanical properties of the aortic annulus by 4-dimensional computed tomography: insights into the functional anatomy of the aortic valve complex and implications for transcatheter aortic valve therapy. J Am Coll Cardiol 59(2):119-127

20. Wuest W, Anders K, Schuhbaeck A et al (2012) Dual source multidetector CT-angiography before transcatheter aortic valve implantation (TAVI) using a high-pitch spiral acquisition mode. Eur Radiol 22(1):51-58

21. Hutter A, Opitz A, Bleiziffer S et al (2010) Aortic annulus evaluation in transcatheter aortic valve implantation. Catheter Cardiovasc Interv 76(7):1009-1019
22. Beeres M, Schell B, Mastragelopoulos A et al (2012) High-pitch dual-source CT angiography of the whole aorta without ECG synchronisation: initial experience. Eur Radiol 22(1):129-137

23. Bertaso AG, Wong DT, Liew GY et al (2012) Aortic annulus dimension assessment by computed tomography for transcatheter aortic valve implantation: differences between systole and diastole. Int $\mathrm{J}$ Cardiovasc Imaging 28(8):2091-2098

24. Achenbach S, Delgado V, Hausleiter J, Schoenhagen P, Min JK, Leipsic JA (2012) SCCT expert consensus document on computed tomography imaging before transcatheter aortic valve implantation (TAVI)/transcatheter aortic valve replacement (TAVR). J Cardiovasc Comput Tomogr 6(6):366-380

25. Lehmkuhl L, Foldyna B, Von Aspern K et al (2013) Inter-individual variance and cardiac cycle dependency of aortic root dimensions and shape as assessed by ECG-gated multi-slice computed tomography in patients with severe aortic stenosis prior to transcatheter aortic valve implantation: is it crucial for correct sizing? Int J Cardiovasc Imaging 29(3):693-703

26. Azzalini L, Sharma UC, Ghoshhajra BB et al (2014) Feasibility of Carm computed tomography for transcatheter aortic valve replacement planning. J Cardiovasc Comput Tomogr 8(1):33-43

27. Thubrikar M, Piepgrass WC, Shaner TW, Nolan SP (1981) The design of the normal aortic valve. Am J Physiol 241(6):H795-H801

28. Wood DA, Tops LF, Mayo JR et al (2009) Role of multislice computed tomography in transcatheter aortic valve replacement. Am J Cardiol 103(9):1295-1301

29. Tops LF, Wood DA, Delgado V et al (2008) Noninvasive evaluation of the aortic root with multislice computed tomography implications for transcatheter aortic valve replacement. JACC Cardiovasc Imaging $1(3): 321-330$

30. Little SH, Shah DJ, Mahmarian JJ (2012) Multimodality noninvasive imaging for transcatheter aortic valve implantation: a primer. Methodist Debakey Cardiovasc J 8(2):29-37

31. Lehmkuhl L, Foldyna B, Haensig M et al (2013) Role of preprocedural computed tomography in transcatheter aortic valve implantation. RöFo 184(10):941-949

32. Zegdi R, Ciobotaru V, Noghin M et al (2008) Is it reasonable to treat all calcified stenotic aortic valves with a valved stent? Results from a human anatomic study in adults. J Am Coll Cardiol 51(5): $579-584$

33. Kodali SK, Williams MR, Smith CR et al (2012) Two-year outcomes after transcatheter or surgical aortic-valve replacement. N Engl J Med 366(18):1686-1695

34. Pasic M, Buz S, Dreysse S et al (2010) Transapical aortic valve implantation in 194 patients: problems, complications, and solutions. Ann Thorac Surg 90(5):1463-1469, discussion 1469-1470

35. Colli A, Nofrerias EF, Escudero A (2011) Aortic root rupture during trans-catheter aortic valve implantation. Eur J Cardiothorac Surg 39(5):788

36. Houthuizen P, van der Boon RM, Urena M et al (2014) Occurrence, fate and consequences of ventricular conduction abnormalities after transcatheter aortic valve implantation. EuroIntervention 9(10): $1142-1150$

37. Gurvitch R, Webb JG, Yuan R et al (2011) Aortic annulus diameter determination by multidetector computed tomography: reproducibility, applicability, and implications for transcatheter aortic valve implantation. JACC Cardiovasc Interv 4(11):12351245 